listing of common tasks, describe which tasks resulted in pain, and share tips for injury prevention.

Results Themes identified through content analysis of transcripts include: a) Care giving relationship-The approach that HCWs take toward their consumers impacts the consumers' behaviours and may reduce injury risks, b) Exchanging information-HCWs want to learn about consumer conditions and assistive devices/ practices to help mitigate pain and improve mobility and c) Problem solving about devices/practices-HCWs have a stake in introducing devices, educating and negotiating with consumers to impact their movement and care.

Conclusions In summary, CHWs have insights into their work and should be supported to 1) identify risky tasks, 2) to document barriers and solutions to implement them, 3) to share resources and 4) to identify training needs. The findings may be explored in labour-management forums in order to create policies and procedures to reduce ergonomic risks to HCWs and their consumers.

\section{THE EFFECTS OF ACUTE AND CHRONIC MENTAL STRESS ON CARDIAC FUNCTION}

Mark Wilson, Lorraine Conroy. University of Illinois at Chicago, Chicago, IL, USA

\subsection{6/oemed-2014-102362.236}

Objectives The main objective of this study is to investigate the effects of chronic and acute stress on blood pressure, heart rate variability and an indicator of cardiac contractility. The study also aims to describe the degree to which various demographic and lifestyle factors modify the observed effect.

Method Chronic stress was assessed with a validated questionnaire, the Perceived Stress Scale. Acute stress was the level of stress experienced from the performance of a simple mental challenge on a scale from 1-100. Heart rate variability is calculated from heart rate measurements recorded via a heart rate monitor. Cardiac contractility index is derived from the amplitude ratio of the first to second heart sounds, as recorded with an electronic stethoscope. Measurements for each of the parameters were taken before and after performance of the task. Pretask measurements were used as baseline data and related to reported chronic stress levels. Acute stress levels were compared with the difference between pre- and post-task cardiac measurements.

Results Average acute stress level was 17/100. A statistically significant increase in cardiac contractility index was found for acute stress, while no significant change in either HRV or heart rate was noted. Subjects with high chronic stress showed a significantly reduced cardiac contractility than those with low chronic stress, while no difference in HRV was found between groups. Conclusions Acute and chronic stress exerts differing inotropic effects on the heart, while no chronotropic effect was observed.

\section{WORKPLACE VIOLENCE AND DEPRESSIVE SYMPTOMATOLOGY AMONG POLICE OFFICER}

${ }^{1}$ Sang Gil Lee, ${ }^{2}$ Inah Kim, ${ }^{3}$ Daeho Kim. 'Occupational Safety and Health Research Institute, Korea Occupational Safety and Health Agency, Incheon, Republic of Korea; ${ }^{2}$ Department of Occupational Health, Graduate School of Public Health, Yonsei University, Seoul, Republic of Korea; ${ }^{3}$ Occupational Lung Diseases Institute, Korea Workers' Compensation and Welfare Service, Gyeonggi-Do, Republic of Korea

10.1136/oemed-2014-102362.237
Objectives The prevalence of depression in police officer is higher than in the general population. Police officers are often exposed to work place violence, long time work, shift work and other pcychosocial stressers. This study was conducted to evaluate the occupational factors associated with depressive symptomatology in police officer.

Method The study sample was 7476 police officers. A structured questionnaire was used to assess exposures to violence, shift working with jobs, health behaviours and sociodemographic factors. Subjects with depressive symptomatology was who experienced depressive symptom for continuously for more than 2 weeks within last 1year. And chronic work place violence exposed subjects was who experienced threat or complaint from work related people more than 4 times per week.

Results Prevalence of depressive symptom among subjects was $35.5 \%(\mathrm{n}=2622)$ and higher in subjects exposed to chronic violence. After adjusting covariates, the odds ratio of depression was 2.01 (95\% CI; 1.80-2.25) for chronic work place violence exposed subjects $(\mathrm{n}=2005,27.11 \%), 1.20$ (95\% CI; $1.02-$ 1.41) for shift working subjects $(n=6270,85.68 \%)$.

Conclusions Our findings support the association workplace violence exposure between depressive symptomatology. Further study with more detailed work place violence exposure classification and measurement is need to confirm the association workplace violence and depressive symptomatology.

\section{THE COMBINED EFFECTS OF HIGH EMOTIONAL DEMANDS AND LOW JOB CONTROL AT WORK ON SUICIDAL IDEATION IN KOREAN SALES AND SERVICE WORKERS}

1,2,3 Jin-Ha Yoon, ${ }^{3,4}$ Sei-Jin Chang. 'Institute for Occupational Health, Yonsei University College of Medicine, Seoul, Korea; ${ }^{2}$ Department of Preventive Medicine, Yonsei University College of Medicine, Seoul, Korea; ${ }^{3}$ Department of Preventive Medicine, Wonju College of Medicine, Yonsei University, Wonju, Korea; ${ }^{4}$ Institute Occupational and Environmental Medicine, Wonju College of Medicine, Yonsei University, Wonju, Korea

\subsection{6/oemed-2014-102362.238}

Objectives Suicide rates have increased worldwide, and suicide is the second leading cause of death below the age of 60 in Korea. Hence, loss of the working years due to suicide is very important issue in occupational health. We examined the effects of high emotional demand and low job control on suicidal ideation in sales and service workers.

Method A total of 1995 participants (824 men and 1171 women) were recruited in this study. Suicidal ideation, high emotional demand and low job control were estimated by self-report questionnaires from the 4th Survey. Odds ratios (OR) and 95\% confidence intervals $(95 \% \mathrm{CI}$ ) for suicidal ideation was calculated by gender using the logistic regression analysis.

Results People who experienced high emotional demands (OR: 2.07 in men, OR: 1.97 in women) or low job control (OR: 1.96 in men, OR: 1.33 in women) were more likely to experience suicidal ideation, after adjusting for age, household income, and employment characteristics (paid vs. self-employed workers). The combined effects of emotional demands and job control revealed that workers with high emotional demand and high job control (OR: 1.93 in men, OR: 1.60 in women) and high emotional demand and low job control (OR: 4.60, OR: 2.69 in women) had a higher risk for suicidal ideation, compared to workers with low emotional demand and high job control, after controlling for age, household income, 
employment characteristics (paid vs. self-employed workers), smoking, alcohol consumption, and physical activity.

Conclusions Our current results suggest that high emotional demand in both genders as well as low job control in men might play a crucial role in increasing the odds of suicidal ideation in sales and services workers. These serious links were still significant after controlling for individual risk factors such as for age, household income, and lifestyle factors. Furthermore, strong additive relationships of combination of high emotional demand with low job control to the odds of suicidal ideation were found both in men and women.

\section{DO PARTICIPANTS WHO COMPLETE A TELEPHONE SURVEY IN A LANGUAGE OTHER THAN ENGLISH DIFFER TO THOSE WHO COMPLETE THE SURVEY IN ENGLISH?}

${ }^{1}$ Terry Boyle, ${ }^{1}$ Renee Carey, ${ }^{1}$ Susan Peters, ${ }^{2}$ Deborah Glass, ${ }^{1}$ Lin Fritschi, ${ }^{1}$ Alison Reid. ${ }^{1}$ The University of Western Australia, Perth, Western Australia, Australia; ${ }^{2}$ Monash University, Melbourne, Victoria, Australia

\subsection{6/oemed-2014-102362.239}

Objectives Limited research indicates that using English-language only surveys in prevalence studies conducted in the general population or in specific ethnic populations may result in unrepresentative samples and biassed results. In this study we investigated whether participants from ethnic minorities who chose to complete a study interview in a language other than English (LOTE) differed from those who completed the interview in English.

Method This study was conducted within the Migrant Australian Workplace Exposure Study, a population-based telephone survey that assessed the prevalence of exposure to occupational carcinogens among 749 workers of Chinese, Vietnamese and Arabic ancestry. The study was conducted in Australia in 2013. Modified Poisson regression determined the demographic factors associated with completing the interview in a LOTE.

Results Participants who completed the interview in a LOTE differed from those who completed the interview in English on several demographic factors, including sex, city of residence and country of birth. They were more likely to have a post-school qualification and to speak a LOTE at home, and were also more likely to be exposed at carcinogens at work compared with those who completed the interview in English (40\% compared with $\left.29 \%, P_{\text {Difference }}<0.01\right)$.

Conclusions The participants who choose to complete the study interview in their native language had several demographic differences to those participants who completed it in English, and were more likely to be exposed to carcinogens at work. Prevalence studies that offer only English-language study instruments are unlikely to produce representative samples of minority groups, and may therefore produce biassed results.

\section{RESPIRATORY SURVEILLANCE IN THAI AUTOMOBILE WORKERS}

Worrapan Karnjanakantorn. Bangkok Hospital Group, Bangkok, Thailand

10.1136/oemed-2014-102362.240
Objectives To survey the pulmonary function test in automobile workers.

Background There are some working processes in automobile factory that can affect lung function. Thailand had developed health surveillance in respiratory e.g. predicted values of pulmonary function test for Thai population for a decade.

Methods A cross-sectional survey was conducted during JuneAugust 2013 among the workers. Data was collected through periodic examination from 165 people who participated in the study.

Results All workers were male. They were 26-54 years of age and their average working years were $8.8 \pm 2.6$ years. The report of abnormal pulmonary function test was $12.7 \%$ (21 people). They were found to have restricted lung function and had mild to moderate severity without any abnormal chest Xray. There was $19 \%$ (4 people) who had abnormal as same as the previous test in 2012 . There was $47 \%$ (10 people) who work in welding and body painting zone. The other biological monitoring 2,5-dioxohexane, Toluene, Xylene was done in 17 people who were exposed to these substances and the level was normal.

Conclusions Pulmonary function test is a useful test especially for health surveillance in welding and painting zone even though occupational or work related lung disease was not diagnosed. The environmental examination should be done to explain the working condition.

\section{THE ASSOCIATION OF BLOOD LEAD LEVELS AND BONE DENSITY IN DIFFERENT COMBINATIONAL SNP POLYMORPHISMS AMONG TAIWAN LEAD WORKERS}

${ }^{1}$ Chien-Juan Chen, ${ }^{1}$ Hsueh-Wei Chang, ${ }^{2}$ Cheng-Hong Yang, ${ }^{1} H u n g-Y i$ Chuang. ${ }^{1}$ Kaohsiung Medical University, Kaohsiung, Taiwan; ${ }^{2}$ National Kaohsiung University of Applied Sciences, Kaohsiung, Taiwan

\subsection{6/oemed-2014-102362.241}

Objectives Past researches almost explored the relationship between a single gene with a single disease. Our study aims to investigate into the interaction of multi-gene with a single disease by using Genetic Algorithms.

Method Samples in this study are from a lead battery factory in Taiwan. We collected the data of their bone density, blood lead levels and 6 SNPs (ACE, alpha-adducin, Bsm, Tag, Apa, ALAD) from 1990 to 2009 . When in 2009, a total of 502 employees in this factory. And we used Genetic Algorithms and logistic regression analysis that the genotype in an individual.

Results In this study, our findings suggest that when people's genotype combined Bsm bb and ALAD 1-1, it will have a protective effect on bone density. It means the Taiwanese lead worker with genotypes of Bsm bb and ALAD 1-1, would have less chance to have low bone density (OR: 0.58 ; 95\% CI: $0.95-$ $0.35)$.

Conclusions We found the results by using Genetic Algorithms and logistic regression analysis that the genotype in an individual which are Bsm bb type and ALAD 1-1 type plays an important role in protecting bone density among 245 male employees and 261 female employees. In conclusion, our study found Bsm gene and ALAD gene influence bone density. However, the mechanism and the exact relationship between two genes and bone density need further investigation. 